\title{
Potential Neuroimmunological Targets in the Treatment of Anxiety Disorders
}

Ruihua Hou a Zhen Tang b D David S. Baldwin a

a University Department of Psychiatry, Clinical and Experimental Sciences, Faculty of Medicine,

University of Southampton, Southampton , UK; ь Suzhou University Guangji Hospital, Suzhou , China

\begin{abstract}
In the translation of psychoneuroimmunology research into clinical practice, one critical step is to identify biomarkers for improved diagnosis and targeting of interventions. Inflammatory markers deserve special attention due to their crucial role linking various health conditions and disorders. In this chapter, authors discuss the pivotal roles of cytokines in signalling to the brain and leading to behavioural changes. This is followed by a review of recent research findings into neuroimmunology of depression, and immunomodulating effects of antidepressants. The rest of the chapter focuses on neuroinflammatory hypothesis in anxiety disorders, and provides an overview of current research evidence on inflammatory responses in anxious state and anxiety disorders. Research suggestions are recommended, including study design, risk factors, medication effects, and measurement strategies. Clinical and pharmacotherapeutic implications and future research directions are also discussed in the final section.

Copyright (C) 2013 S. Karger AG, Basel
\end{abstract}

